\title{
The role of gender inequities in women's access to reproductive health care: a population-level study of Namibia, Kenya, Nepal, and India
}

This article was published in the following Dove Press journal:

International Journal of Women's Health

26 July 2012

Number of times this article has been viewed

\author{
Amrita Namasivayam' \\ Donatus C Osuorah ${ }^{2}$ \\ Rahman Syed ${ }^{3}$ \\ Diddy Antai ${ }^{3,4}$ \\ 'Department of Public Health, \\ Division of Global Health (IHCAR), \\ Karolinska Institute, Stockholm, \\ Sweden; 'Department of Pediatrics, \\ Nnamdi Azikiwe University \\ Teaching Hospital, Nnewi, Anambra, \\ Nigeria; ${ }^{3}$ Department of Public \\ Health, Division of Social Medicine, \\ Karolinska Institute, Stockholm, \\ Sweden; ${ }^{4}$ Division of Global Health \\ and Inequalities, The Angels Trust, \\ Abuja, Nigeria
}

Background: The role of gender inequities in explaining women's access to reproductive health care was examined in four countries (two sub-Saharan African and two South Asian countries). The extent of gender inequities varies across and within countries, and is rooted in the different cultural practices and gender norms within these different countries, and differences in the status and autonomy of women.

Methods: Demographic and Health Survey data from women aged 15-49 years within these countries were analyzed with multivariate logistic regression analysis to examine the role of multidimensional characteristics of gender inequities, operationalized as access to skilled antenatal care, tetanus toxoid injection during pregnancy, and access to skilled antenatal care.

Results: Significant associations were found between several dimensions of gender inequities (with the exception of decision-making autonomy) and reported use of maternal reproductive health care services. Several pathways of influence between the outcome and exposure variables were also identified.

Conclusion: Dimensions of gender inequities (with the exception of decision-making autonomy) differentially influenced woman's use of reproductive health care services, thus highlighting the urgent need for concerted and sustained efforts to change these harmful traditional values if several of these countries are to meet Millennium Development Goal-5.

Keywords: women, gender inequities, reproductive health care, Namibia, Kenya, Nepal, India

\section{Introduction}

The World Health Organization estimated in 2011 that 358,000 women die every year during pregnancy and childbirth, ${ }^{1}$ often from preventable causes. These deaths occur mostly due to lack of access to skilled health care and emergency services during pregnancy and immediately after childbirth, as well as lack of contraception needed to avoid unwanted pregnancies as a means of birth spacing. ${ }^{2}$ About $99 \%$ of all maternal deaths occur in low- and middle-income countries. ${ }^{3}$ More than half of these deaths occur in sub-Saharan Africa (SSA), and one-third in South Asia; together, these regions account for $87 \%$ of all maternal deaths. ${ }^{4}$ These figures are in spite of the remarkable progress many of these countries have made in reducing maternal mortality rates following implementation of such policies as the Alma Ata Declaration in 1978, the Safe Motherhood initiative initiated by the World Bank in 1987, 5,6 and more recently, the Millennium Development Goals (MDGs). ${ }^{7}$ MDG-5 aims to reduce maternal mortality ratios by $75 \%$, between 1990 and 2015, and achieve universal reproductive health services for women by 2015 . The provision of maternal health care services has been
Correspondence: Diddy Antai Department of Public Health, Division of Social Medicine, Karolinska Institute, I7I 77 Stockholm, Sweden

Tel +4687373946

Fax +468334693

Email diddy.antai@ki.se 
repeatedly shown to be essential in curbing maternal deaths. ${ }^{8,9}$ Factors such as access to antenatal care, ${ }^{10,11}$ skilled assistance at child birth, ${ }^{12,13}$ delivery at a health care facility, access to emergency obstetric care, ${ }^{14}$ and appropriate postpartum care ${ }^{15}$ have been effective in reducing maternal mortality.

\section{Regional contexts: SSA and South Asia}

The problem of maternal mortality in SSA has long been acknowledged. With a lifetime risk of maternal death of one in $31,{ }^{16} \mathrm{SSA}$ has not adequately met the goal of reducing maternal mortality by $75 \%$ in the last decade. ${ }^{17}$ Many countries in SSA have had to deal with the human immunodeficiency virus/acquired immunodeficiency syndrome epidemic on a much larger scale than anywhere else in the world, ${ }^{18}$ thus accounting for $9 \%$ of all maternal deaths. ${ }^{19}$ These poor figures have been attributed to factors such as lack of skilled attendance at birth, maternal illiteracy, poor socioeconomic status, and high fertility rates, as well as national-level factors such as health expenditure per capita and the political stability of the country. ${ }^{20,21}$ In South Asia, the lifetime maternal mortality risk of one in 120 is only second to that of SSA. India alone accounts for 63,000 maternal deaths a year - the largest number in any country. ${ }^{16}$ Progress toward achieving MDG-5 in this region has also been slow, ${ }^{22}$ and - as in the case of SSA - one of the principal contributing factors is the lack of skilled maternal health care. ${ }^{23}$ Additional factors, however, include inequality in health care access mainly due to economic, social, religious, or ethnic stratification, ${ }^{23}$ delays in seeking and obtaining health care, ${ }^{24}$ and poor quality of care due to a lack of skilled health care professionals. ${ }^{25}$ In many South Asian countries, a major obstacle preventing women from receiving the health care they need is out-ofpocket payments. ${ }^{26}$

\section{The role of gender inequities}

Conceptually distinct from "sex," which is biological and involving the ability to bear a child, gender is a social construct consisting of gender roles, norms, and expectations attributed to men and women in a given society; ${ }^{27}$ this varies between societies or era. Gender plays a role in the access to and use of maternal health care on several levels. Gender norms/roles are often determined by culture and the societies in which people live, and vary significantly across and within cultures; these norms also govern differences in roles, rights, and opportunities for men and women in that society. ${ }^{28}$ Gender inequities refer to the discrimination and differential treatment of men or women in ways that are unfair, avoidable, unjust, and/or unnecessary. ${ }^{29}$ In societies where women are of a lower status than men, gender inequities are often mirrored in terms of restrictions in education, health care, economic and employment opportunities, and choices regarding marriage and reproductive health matters. ${ }^{30} \mathrm{MDG}-3$ aims to "promote gender equality and empower women," with specific focus on eliminating barriers to education and employment and rights to health care. ${ }^{31}$

Gender inequities are multidimensional and affect women's access to health care in more ways than one. ${ }^{32}$ Women generally have higher life expectancies than men, due to biological and behavioral factors. Yet this advantage is overridden in many contexts, and female life expectancy at birth is sometimes lower than or equal to that of males. ${ }^{33}$ Additionally, women's greater longevity often does not translate into healthier lives, and in many low- and middle-income countries, women undergoing pregnancy and childbirth are often unable to access maternal health care due to systematic discriminations or inequities rooted in gender norms within the society they live in. Lack of autonomy, male dominance in relationships, and gender-based violence are other examples of gender inequities that affect access to health care. ${ }^{27,33}$

\section{Conceptual framework on how gender inequities affect access to maternal health care services}

This study employs a conceptual framework adapted from the Ecological Systems Theory ${ }^{34}$ in investigating the association between women's access to reproductive health care and gender inequities at three different levels (individual, relationship, and societal), both of which are multidimensional and interlinked. The status of women and gender norms in a society determine to a great extent the dynamics of women's relationships with their spouses or male partners, and their place in the household. Societal factors also affect the opportunities woman have regarding education, income, and occupation, expected age at first marriage, her control over earnings, and participation in decision making. The reverse could also hold true: lower educational opportunities, lack of decision-making autonomy and control over earnings, and the early marriage of women in a society act to reinforce the status quo of relationships in that society, which in turn could maintain women's lower status and the existing gender norms in a society. Societal and community level factors can affect women's access to maternal health care through different pathways (Figure 1): the type of society (eg, patriarchal, traditional) a woman lives in ${ }^{27}$ - and the gender norms and values within the society ${ }^{35}$ - determine her status within the community and household, thereby influencing their access 


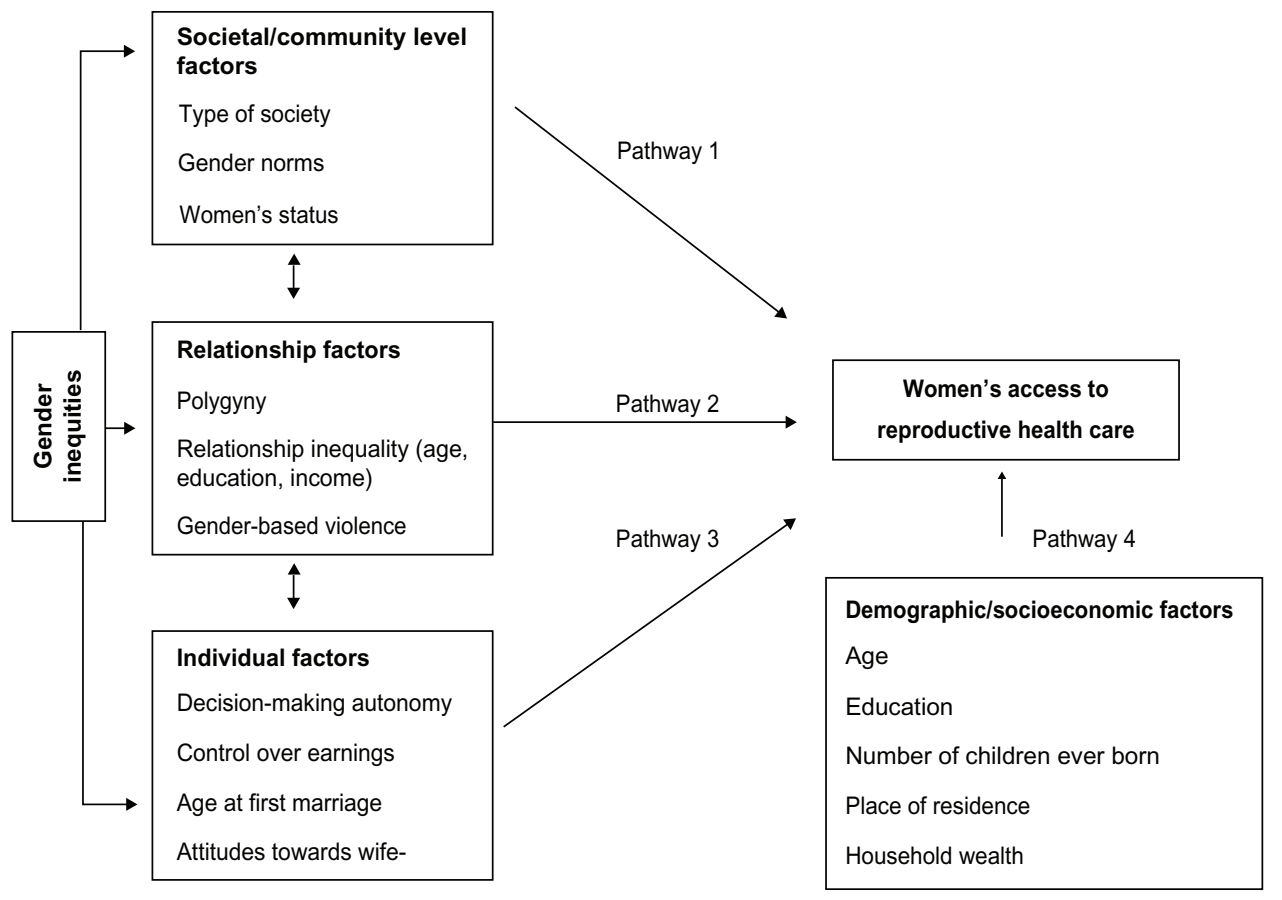

Figure I Conceptual framework.

to health care (Pathway 1). The dynamics of the relationship between a woman and her partner can determine a woman's access to health care. In patriarchal societies, polygyny is a common practice, and inequalities between a woman and her partner in terms of age, education, and income often result in women being in positions of dependency with little or no say in matters such as health care, often needing to seek permission from their partners or mother-in-law to carry out daily activities. ${ }^{10,36}$ Varying levels of partner controlling behaviors (including gender-based violence) have been shown to affect women's access to health care (Pathway 2). ${ }^{37}$ Individual-level factors such as a woman's decision-making autonomy, control over earnings, age at first marriage, and attitudes toward wife beating are indicators of empowerment, the degree of dependence on their partners, and the likelihood of access to maternal health care (Pathway 3). ${ }^{35,38}$ Demographic and socioeconomic factors such as a woman's age, education, parity, household wealth, and place of residence can also affect access to health care, both in terms of physical and financial accessibility (Pathway 4). ${ }^{36}$

Therefore, the aim of this study was to assess, from a holistic perspective, the role of gender inequities on women's access to reproductive health care in four countries: India, Nepal, Kenya, and Namibia. It was hypothesized that dimensions of gender inequities would differentially influence access to measures of reproductive health care depending on the social contexts within the countries in the study.

\section{Methods Data}

Four countries, two in SSA (Kenya and Namibia) and two in South Asia (India and Nepal), were selected on the basis of available indicators for measuring exposures and relevant outcomes, and to allow for the comparison of geographic variations in health care utilization within different contexts. Data came from the Demographic and Health Surveys (DHS) conducted in Kenya (2008-2009), Namibia (2006-2007), India (2005-2006), and Nepal (2006). DHS data are nationally representative population-based surveys carried out using a stratified two-stage cluster sampling design. Respondents included 8444 women in Kenya, 9804 women in Namibia, 124,385 women in India, and 10,793 women in Nepal, all between the ages of 15-49 years. Data were collected using a standardized DHS questionnaire administered during faceto-face interviews. Response rates ranged from $94.5 \%$ (India) to $98.4 \%$ (Nepal).

\section{Measures}

Women's access to reproductive health care was operationalized as three outcome variables: (1) access to skilled antenatal care, a dichotomous variable grouped as "yes" (if women received antenatal care from a doctor, nurse, midwife, or from all three) or "no" (if women did not receive antenatal care from a doctor, nurse, or midwife); (2) tetanus toxoid injection during pregnancy, a dichotomous a variable grouped 
as "yes" (if women received one or more tetanus injections before childbirth) or "no" (if women did not receive tetanus injections before childbirth); and (3) access to an institutional delivery, for which responses were categorized as "home" (if respondent delivered in own home/parent's home/someone else's home) or "institutional" (if respondent delivered in government hospital/government health clinic/other public or rural hospital/private hospital/maternity clinic/other private clinic/nongovernmental organization/Red Cross/other).

Dimensions of gender inequities were the main exposure variables, which reflected context-specific multidimensions of gender inequities, and included: (1) "decision-making autonomy," created from dichotomous responses ("yes" or "no") to questions about the respondent having the "final say" regarding own health care, making large household purchases, making household purchases for daily needs, visits to family or relatives, food to be cooked every day, and deciding what to do with the money her husband earns. Responses of "yes" to one or more of these questions were grouped as "respondent has decision-making autonomy" and responses of "no" to all of the statements were grouped as "respondent has no decision-making autonomy;" (2) "husband has other wives," created from the variable "number of other wives respondent's husband has," and categorized as "yes" ("one or more other wives") or "no" ("no other wives"); (3) "justifies wife beating," dichotomous responses ("yes" or "no") created from responses to questions on whether respondents would justify "wife beating" for offences such as going out without telling husband/partner, neglecting the children, arguing with husband/partner, refusing to have sex with husband/ partner, and burning the food. Responses of "no" to all of the statements formed one-half of the dichotomy, responses of "yes" to one or more of the questions formed the other half; (4) "age at first marriage," categorized as $\leq 19$ years and $\geq 20$ years; (5) "control over earnings," binary variable created from responses to the question "who decides how to spend money;" responses of "respondent alone," "respondent and husband/partner," and "respondent and other person" were coded "yes," and responses of "husband/partner alone" and "someone else" were coded "no;" and (6) "relationship inequality," assessed on the basis of differences in age, education, and earnings between the respondent and her husband/ partner. Age inequalities were categorized as "respondent is younger than husband/partner," "same age as husband/ partner," and "older than husband/partner." Education inequalities were categorized as "respondent is less educated than partner," "same education as husband/partner," and "more educated than husband/partner." Earning inequalities were categorized as "respondent earns less than husband/partner," "earns the same as husband/partner," and "earns more than husband/partner."

\section{Demographic and socioeconomic characteristics}

These included: (1) age of respondent (15-24, 25-34, and $>35$ years); (2) highest educational attainment (no education, primary, and secondary or higher); (3) number of children ever born (one or two, three or four, and five or more), (4) place of residence (urban and rural); and (5) household wealth index, constructed from responses to questions about household possession of durable items (eg, radio, refrigerator, television, motorcar) and quality of dwelling (eg, floor type, roof type), using principal component analysis. The resulting index was then categorized (poor, middle, and rich). A summary of the exposure variables used in this study are described in Table 1.

\section{Statistical analysis}

Distribution of the measures of gender inequities, and demographic and socioeconomic characteristics for the four countries were analyzed. The same exposure variables were entered into the models for all four countries. Multivariate logistic regression analysis was used to determine measures of gender inequities significant in predicting women's access to maternal health care. Two models were used in analyzing the data: model 1 (crude model) assessed only measures of gender inequities as predictor variables for women's access to maternal health care, whilst model 2 (adjusted model) included the demographic and socioeconomic characteristics. Measures of association are presented as odds ratios (OR) and $95 \%$ confidence intervals $(95 \% \mathrm{CI})$. Missing data, which were very few, were excluded from the analysis. Data was analyzed using IBM ${ }^{\circledR}$ SPSS version 18.0 (SPSS Inc, Chicago, IL).

\section{Ethical considerations}

As a part of the DHS survey methodology, informed consent was obtained from all participants prior to their participation in the survey, and the collection of information was done confidentially. Permission for the use of datasets in the present study was obtained from Measure DHS (Calverton, MD).

\section{Results}

As shown in Table 2, the women were similar with regards to proportions in decision-making autonomy, husband having 
Table I Summary of definitions and measure of variables used in the study

\begin{tabular}{|c|c|}
\hline Variables & Measures \\
\hline \multicolumn{2}{|l|}{ Dimensions of gender inequities } \\
\hline Decision-making autonomy & Yes; no \\
\hline Husband has other wives (polygyny) & Yes; no \\
\hline Justifies wife beating & Yes; no \\
\hline Age at first marriage & $\leq 19$ years; $\geq 20$ years \\
\hline Control over earnings & Yes; no \\
\hline \multirow[t]{6}{*}{ Age inequalities } & Respondent younger than \\
\hline & husband/partner \\
\hline & Respondent same age as \\
\hline & husband/partner \\
\hline & Respondent older than \\
\hline & husband/partner \\
\hline \multirow[t]{6}{*}{ Education inequalities } & Respondent less educated than \\
\hline & husband/partner \\
\hline & Respondent is as educated as \\
\hline & husband/partner \\
\hline & Respondent more educated \\
\hline & than husband/partner \\
\hline \multirow[t]{6}{*}{ Earning inequalities } & Respondent earns less than \\
\hline & husband/partner \\
\hline & Respondent earns same as \\
\hline & husband/partner \\
\hline & Respondent earns more than \\
\hline & husband/partner \\
\hline \multicolumn{2}{|c|}{ Demographic and socioeconomic factors } \\
\hline Age of respondent & $15-24 ; 25-34 ;>35$ years \\
\hline Educational attainment & $\begin{array}{l}\text { No education; primary; } \\
\text { secondary or higher }\end{array}$ \\
\hline Number of children ever born & $\mathrm{I}-2 ; 3-4 ; \geq 5$ children \\
\hline Place of residence & Urban; rural \\
\hline Wealth index & Poor; middle; rich \\
\hline
\end{tabular}

other wives, relationship inequality in age, education and earning, age of respondent, number of children ever born, place of residence, and wealth index. The proportion of women, however, differed with regard to a higher proportion not justifying wife beating (Namibia: 5612 of $9101,62 \%$; India: 64,266 of 116,999, 55\%; and Nepal: 8146 of 10,724, $76 \%$ ), a higher proportion being $\geq 20$ years at first marriage (Namibia: 2748 of 4259, 65\%), and a higher proportion were $\leq 19$ years (Kenya: 3617 of 5904, 61\%; India: 66,894 of 93,724, 71\%; and Nepal: 7287 of 8640, 84\%).

Of the 2355 respondents in Kenya, 2173 (92\%) reported having control of earnings, with similar results in Namibia (1334 of 1516, 88\%) and India (19,827 of 23,033, 86\%); in contrast, 1919 (86\%) of 2225 women did not have control over earnings in Nepal. More women were $\geq 35$ years in Kenya (4933 of 8444, 58\%), whilst the majority were 15-24 years of age in Namibia (4081 of 9804, 42\%), India (46,762 of $124,385,37 \%)$, and Nepal (4479 of 10,793, 41\%). Women with secondary or higher education were of higher proportion in Namibia (6413 of 9804, 65\%) and India $(66,848$ of $124,373,54 \%)$; in contrast, women with primary education were of higher proportion in Kenya (4404 of 8444, 52\%), and women with no education were of higher proportion in Nepal (5677 of 10,793, 52\%) (Table 2).

\section{Factors associated with the outcome measures}

Results of the adjusted analyses are presented in Tables 3-5.

\section{Skilled antenatal care}

Adjusted analyses indicated that among the dimensions of gender inequities, education inequality, ie, women more educated than their partner in Namibia (OR: 0.15; 95\% CI: $0.02-0.99)$ and women with less education than partner in Nepal (OR: 0.46; 95\% CI: $0.28-0.75$ ), were less likely to access skilled antenatal care compared to women with the same level of education as their partner. In contrast, the likelihood of having access to skilled antenatal care was significantly higher for women who were $\leq 19$ years at first marriage in Nepal (OR: 1.72; 95\% CI: 1.08-2.73) compared to being aged $\geq 20$ years, among women with less education than their partner in India (OR: 1.26; 95\% CI: 1.10-1.45) compared to having the same level of education as their partner, and among women earning less than their partner in India (OR: $1.48 ; 95 \% \mathrm{CI}: 1.22-1.80$ ) compared to earning the same as their partner.

In addition, access to skilled antenatal care was significantly less likely for women aged 15-24 years in Namibia (OR: 0.07 ; 95\% CI: 0.01-0.64) compared to those $\geq 35$ years, but access to skilled antenatal care was significantly more likely for women having no education (OR: 0.09; $95 \%$ CI: $0.03-0.25$ ) or primary education (OR: 0.43 ; $95 \%$ CI: $0.19-0.98$ ) in Kenya, having no education (OR: $0.39 ; 95 \%$ CI: $0.32-0.49)$ or primary education (OR: 0.66 ; $95 \% \mathrm{CI}$ : $0.52-0.84$ ) in India, and having no education (OR: 0.46; 95\% CI: $0.28-0.75$ ) in Nepal compared to having secondary or higher education. Women who had three or four children in India (OR: 0.55; 95\% CI: $0.47-0.65$ ) and Nepal (OR: 0.58 ; $95 \%$ CI: $0.37-0.89)$ as well as those with five or more children in India (OR: 0.33; 95\% CI: $0.27-0.40$ ) were less likely to have access to skilled antenatal care compared to women who had one or two children. Women residing in rural areas in India (OR: 0.69; 95\% CI: $0.58-0.82$ ) and Nepal (OR: 0.26 ; 95\% CI: $0.18-0.37$ ) had a significantly lower likelihood of having access to skilled antenatal care 
Table 2 Characteristics of the women from Kenya, Namibia, India, and Nepal in the study

\begin{tabular}{|c|c|c|c|c|}
\hline \multirow[t]{2}{*}{ Variables } & \multicolumn{4}{|l|}{ Countries } \\
\hline & $\begin{array}{l}\text { Kenya } \\
(\mathrm{N}=\mathbf{8 4 4 4})\end{array}$ & $\begin{array}{l}\text { Namibia } \\
(N=9804)\end{array}$ & $\begin{array}{l}\text { India } \\
(N=\mid 24,385)\end{array}$ & $\begin{array}{l}\text { Nepal } \\
(N=10,793)\end{array}$ \\
\hline Decision-making autonomy & $n=4788$ & $n=3332$ & $\mathrm{n}=85,943$ & $\mathrm{n}=10,793$ \\
\hline Yes & $4660(97 \%)$ & 3070 (92\%) & 76,017 (88\%) & $6164(57 \%)$ \\
\hline No & $128(3 \%)$ & $262(8 \%)$ & 9926 (12\%) & $4629(43 \%)$ \\
\hline Husband has other wives & $n=504 I$ & $\mathrm{n}=3562$ & $\mathrm{n}=87,925$ & $\mathrm{n}=8297$ \\
\hline Yes & $885(18 \%)$ & $649(18 \%)$ & $1980(2 \%)$ & 385 (5\%) \\
\hline No & $4156(82 \%)$ & $2913(82 \%)$ & $85,945(98 \%)$ & 7912 (95\%) \\
\hline Justifies wife beating & $\mathrm{n}=7975$ & $\mathrm{n}=9010$ & $n=116,999$ & $\mathrm{n}=10,724$ \\
\hline Yes & 3985 (50\%) & 3398 (38\%) & $52,733(45 \%)$ & $2578(24 \%)$ \\
\hline No & 3990 (50\%) & $5612(62 \%)$ & $64,266(55 \%)$ & $8146(76 \%)$ \\
\hline Age at first marriage & $\mathrm{n}=5904$ & $\mathrm{n}=4259$ & $\mathrm{n}=93,724$ & $\mathrm{n}=8640$ \\
\hline$\leq 19$ years & $3617(61 \%)$ & $151 \mid(35 \%)$ & $66,894(71 \%)$ & 7287 (84\%) \\
\hline$\geq 20$ years & 2287 (39\%) & 2748 (65\%) & $26,830(29 \%)$ & $1353(16 \%)$ \\
\hline Control over earnings & $\mathrm{n}=2355$ & $\mathrm{n}=1516$ & $\mathrm{n}=23,033$ & $\mathrm{n}=2225$ \\
\hline Yes & $2173(92 \%)$ & I 334 (88\%) & 19,827 (86\%) & $306(14 \%)$ \\
\hline No & $182(8 \%)$ & $182(12 \%)$ & $3206(14 \%)$ & $1919(86 \%)$ \\
\hline Age inequality & $\mathrm{n}=5021$ & $\mathrm{n}=3446$ & $\mathrm{n}=88,078$ & $\mathrm{n}=8243$ \\
\hline Respondent is younger than partner & $4734(94 \%)$ & $2823(82 \%)$ & $83,127(94 \%)$ & $7107(86 \%)$ \\
\hline Respondent is same age as partner & $144(3 \%)$ & $206(6 \%)$ & $2632(3 \%)$ & $572(7 \%)$ \\
\hline Respondent is older than partner & $143(3 \%)$ & $408(12 \%)$ & $2319(3 \%)$ & $564(7 \%)$ \\
\hline Education inequality & $\mathrm{n}=5809$ & $\mathrm{n}=3989$ & $n=93,199$ & $\mathrm{n}=8652$ \\
\hline Respondent is less educated than partner & $270 \mathrm{I}(46 \%)$ & 1549 (39\%) & $48,506(52 \%)$ & $5503(64 \%)$ \\
\hline Respondent is as educated as partner & $1843(32 \%)$ & $980(24 \%)$ & $28,252(30 \%)$ & $2559(29 \%)$ \\
\hline Respondent is more educated than partner & $1265(22 \%)$ & $1460(37 \%)$ & $|6,44|(18 \%)$ & $590(7 \%)$ \\
\hline Earning inequality & $\mathrm{n}=6154$ & $\mathrm{n}=1486$ & $n=22,610$ & $\mathrm{n}=2202$ \\
\hline Respondent earns less than partner & $|53|(67 \%)$ & $102 \mid(69 \%)$ & $|6,75|(74 \%)$ & $1904(68 \%)$ \\
\hline Respondent earns the same as partner & $388(17 \%)$ & $174(12 \%)$ & $2678(12 \%)$ & $547(25 \%)$ \\
\hline Respondent earns more than partner & $371(16 \%)$ & $291(19 \%)$ & $3181(14 \%)$ & $151(7 \%)$ \\
\hline Age of respondent & $\mathrm{n}=8444$ & $\mathrm{n}=9804$ & $\mathrm{n}=124,385$ & $\mathrm{n}=10,793$ \\
\hline 15-24 years & $35 I I(4 I \%)$ & $408 \mathrm{I}(42 \%)$ & $46,762(37 \%)$ & $4479(41 \%)$ \\
\hline $25-34$ years & $2603(31 \%)$ & $2985(30 \%)$ & $38,520(31 \%)$ & $3101(29 \%)$ \\
\hline$\geq 35$ years & $4933(58 \%)$ & $2738(28 \%)$ & $39,103(32 \%)$ & $3213(30 \%)$ \\
\hline Highest educational level & $\mathrm{n}=8444$ & $\mathrm{n}=9804$ & $\mathrm{n}=124,373$ & $\mathrm{n}=10,793$ \\
\hline No education & $1242(15 \%)$ & $775(8 \%)$ & $39,769(32 \%)$ & $5677(52 \%)$ \\
\hline Primary & $4404(52 \%)$ & $2616(27 \%)$ & $17,756(14 \%)$ & $1908(18 \%)$ \\
\hline Secondary or higher & $2798(33 \%)$ & $6413(65 \%)$ & $66,848(54 \%)$ & $3208(30 \%)$ \\
\hline Number of children ever born & $\mathrm{n}=6102$ & $\mathrm{n}=6636$ & $\mathrm{n}=84,609$ & $\mathrm{n}=7791$ \\
\hline $1-2$ & 2393 (39\%) & 3494 (53\%) & $39,698(47 \%)$ & $3153(41 \%)$ \\
\hline $3-4$ & $1825(30 \%)$ & $1869(28 \%)$ & $30,150(36 \%)$ & 2679 (34\%) \\
\hline$\geq 5$ & $1884(31 \%)$ & $1273(19 \%)$ & $|4,76|(\mid 7 \%)$ & $1959(25 \%)$ \\
\hline Place of residence & $\mathrm{n}=8444$ & $\mathrm{n}=9804$ & $\mathrm{n}=124,385$ & $\mathrm{n}=10,793$ \\
\hline Urban & 2615 (31\%) & 4405 (45\%) & $56,96 \mid(46 \%)$ & 2949 (27\%) \\
\hline Rural & 5829 (69\%) & 5399 (55\%) & $67,424(54 \%)$ & 7844 (73\%) \\
\hline Wealth index & $\mathrm{n}=8444$ & $\mathrm{n}=9804$ & $n=124,385$ & $\mathrm{n}=10,793$ \\
\hline Poor & 2983 (35\%) & 3350 (34\%) & 31,729 (25\%) & 4212 (39\%) \\
\hline Middle & 1455 (I7\%) & $2223(23 \%)$ & $23,682(19 \%)$ & $1974(18 \%)$ \\
\hline Rich & $4006(48)$ & 423 I (43\%) & $68,974(56 \%)$ & 4607 (43\%) \\
\hline
\end{tabular}

compared to women residing in urban areas. Finally, women in the poorest (OR: 0.25 ; 95\% CI: $0.19-0.31$ ) and middle (OR: 0.45 ; 95\% CI: $0.35-0.58$ ) wealth categories in India and women in the poorest (OR: $0.40 ; 95 \% \mathrm{CI}: 0.28-0.58$ ) and middle (OR: 0.62; 95\% CI: 0.39-0.97) wealth categories in Nepal had a significantly lower likelihood of access skilled antenatal care compared to women in the richest wealth category (Table 3 ).

\section{Access to institutional delivery}

Access to institutional delivery was significantly less likely among women whose husband had other wives in Nepal (OR: 
Table 3 Odds ratios and $95 \%$ confidence intervals for the association between measures of gender inequities and women's access to skilled antenatal care

\begin{tabular}{|c|c|c|c|c|}
\hline \multirow[t]{4}{*}{ Variables } & \multicolumn{4}{|l|}{ Countries } \\
\hline & Kenya & Namibia & India & Nepal \\
\hline & Adjusted & Adjusted & Adjusted & Adjusted \\
\hline & OR (95\% Cl) & OR (95\% Cl) & OR $(95 \% \mathrm{Cl})$ & OR $(95 \% \mathrm{CI})$ \\
\hline \multicolumn{5}{|l|}{ Gender inequities } \\
\hline \multicolumn{5}{|l|}{ Decision-making autonomy } \\
\hline Yes & I & 1 & 1 & I \\
\hline No & $0.53(0.06-4.79)$ & $1.45(0.10-20.47)$ & $0.89(0.7 I-I . I I)$ & $0.86(0.54-1.36)$ \\
\hline \multicolumn{5}{|l|}{ Husband has other wives } \\
\hline Yes & $1.14(0.63-2.04)$ & $1.39(0.24-7.94)$ & $0.82(0.58-1.16)$ & $0.65(0.33-1.29)$ \\
\hline No & $\mathrm{I}$ & I & I & I \\
\hline \multicolumn{5}{|l|}{ Justifies wife beating } \\
\hline Yes & $1.15(0.07-1.88)$ & $0.81(0.26-2.52)$ & $0.97(0.85-1.10)$ & $0.80(0.57-1.13)$ \\
\hline No & 1 & 1 & 1 & 1 \\
\hline \multicolumn{5}{|l|}{ Age at first marriage } \\
\hline$\leq 19$ years & $0.7 \mid(0.40-1.24)$ & $0.84(0.25-2.85)$ & $0.84(0.69-1.01)$ & $1.72(1.08-2.73)$ \\
\hline$\geq 20$ years & 1 & 1 & 1 & 1 \\
\hline \multicolumn{5}{|l|}{ Control over earnings } \\
\hline Yes & 1 & 1 & 1 & I \\
\hline No & $1.35(0.54-3.4 I)$ & $1.25(0.19-8.07)$ & $1.18(0.98-1.42)$ & I.II (0.69-I.77) \\
\hline \multicolumn{5}{|l|}{ Relationship inequality } \\
\hline \multicolumn{5}{|l|}{ Age inequality } \\
\hline Younger than partner & $0.98(0.2 \mathrm{I}-4.47)$ & $0(0)^{\S}$ & $1.36(0.96-1.92)$ & I.7I (0.86-3.39) \\
\hline Same age as partner & I & 1 & I & I \\
\hline Older than partner & $0.46(0.07-3.19)$ & $0(0)^{\S}$ & $1.21(0.74-1.98)$ & $1.04(0.43-2.50)$ \\
\hline \multicolumn{5}{|l|}{ Education inequality } \\
\hline Less educated than partner & $1.60(0.95-2.72)$ & $0.74(0.14-3.98)$ & $1.26(1.10-1.45)$ & $0.46(0.28-0.75)$ \\
\hline Same education as partner & 1 & 1 & 1 & 1 \\
\hline More educated than partner & $1.34(0.67-2.67)$ & $0.15(0.02-0.99)$ & $1.14(0.89-1.47)$ & $0.72(0.43-1.22)$ \\
\hline \multicolumn{5}{|l|}{ Earning inequality } \\
\hline Earns less than partner & $0.87(0.46-|.7|)$ & $0.40(0.07-2.17)$ & $1.48(1.22-1.80)$ & $0.91(0.62-1.35)$ \\
\hline Earns the same as partner & I & I & I & I \\
\hline Earns more than partner & $1.24(0.5 \mathrm{I}-3.00)$ & $1.42(0.10-19.58)$ & I.3I (0.99-I.72) & $0.48(0.22-1.02)$ \\
\hline \multicolumn{5}{|l|}{ Age of respondent } \\
\hline $15-24$ years & $1.93(0.76-4.93)$ & $0.07(0.01-0.64)$ & $1.03(0.80-1.32)$ & $1.24(0.64-2.43)$ \\
\hline $25-34$ years & $1.09(0.62-1.92)$ & $0.67(0.17-2.66)$ & $1.16(0.97-1.40)$ & $1.21(0.72-2.02)$ \\
\hline$\geq 35$ years & 1 & 1 & 1 & 1 \\
\hline \multicolumn{5}{|l|}{ Highest educational level } \\
\hline No education & $0.09(0.03-0.25)$ & $0.24(0.04-1.57)$ & $0.39(0.32-0.49)$ & $0.46(0.28-0.75)$ \\
\hline Primary & $0.43(0.19-0.98)$ & $3.20(0.72-14.35)$ & $0.66(0.52-0.84)$ & $0.72(0.43-1.22)$ \\
\hline Secondary or higher & 1 & 1 & 1 & 1 \\
\hline \multicolumn{5}{|l|}{ Number of children ever born } \\
\hline $1-2$ & I & 1 & I & 1 \\
\hline $3-4$ & $1.27(0.59-2.74)$ & $0.22(0.04-1.10)$ & $0.55(0.47-0.65)$ & $0.58(0.37-0.89)$ \\
\hline$\geq 5$ & $0.74(0.32-1.72)$ & $0.15(0.02-1.20)$ & $0.33(0.27-0.40)$ & $0.65(0.37-1.17)$ \\
\hline \multicolumn{5}{|l|}{ Place of residence } \\
\hline Urban & 1 & 1 & 1 & 1 \\
\hline Rural & $0.49(0.22-1.14)$ & $0.29(0.07-1.26)$ & $0.69(0.58-0.82)$ & $0.26(0.18-0.37)$ \\
\hline \multicolumn{5}{|l|}{ Wealth index } \\
\hline Poor & $0.70(0.34-1.43)$ & $0.21(0.04-1.03)$ & $0.25(0.19-0.31)$ & $0.40(0.28-0.58)$ \\
\hline Middle & $0.79(0.37-1.73)$ & $0.42(0.09-1.88)$ & $0.45(0.35-0.58)$ & $0.62(0.39-0.97)$ \\
\hline Rich & 1 & 1 & I & 1 \\
\hline
\end{tabular}

Note: Bold figures indicate statistical significance.

Abbreviations: $\mathrm{Cl}$, confidence interval; $\mathrm{OR}$, odds ratio.

0.17; 95\% CI: $0.05-0.63)$ compared to women who were the only wife, among women who justified wife beating in Namibia (OR: 0.50; 95\% CI: 0.26-0.99) compared to those who did not justify wife beating, and among women who were $\leq 19$ years at first marriage in Kenya (OR: 0.61; 95\%
CI: $0.45-0.82)$ and India (OR: 0.67; 95\% CI: 0.58-0.79) compared to being $\geq 20$ years at first marriage. Furthermore, institutional delivery was less likely among women younger than their partner in Kenya (OR: 0.85; 95\% CI: 0.36-2.04) and among women more educated than their partner in Kenya 
Table 4 Odds ratios and 95\% confidence intervals for the association between measures of gender inequities and women's access to institutional delivery

\begin{tabular}{|c|c|c|c|c|}
\hline \multirow[t]{4}{*}{ Variables } & \multicolumn{4}{|l|}{ Countries } \\
\hline & Kenya & Namibia & India & Nepal \\
\hline & Adjusted & Adjusted & Adjusted & Adjusted \\
\hline & OR $(95 \% \mathrm{Cl})$ & OR $(95 \% \mathrm{Cl})$ & OR $(95 \% \mathrm{Cl})$ & OR $(95 \% \mathrm{Cl})$ \\
\hline \multicolumn{5}{|l|}{ Gender inequities } \\
\hline \multicolumn{5}{|l|}{ Decision-making autonomy } \\
\hline Yes & 1 & I & I & 1 \\
\hline No & $0.58(0.12-2.89)$ & $1.49(0.35-6.29)$ & $0.8 \mathrm{I}(0.65-1.02)$ & $1.13(0.65-1.96)$ \\
\hline \multicolumn{5}{|l|}{ Husband has other wives } \\
\hline Yes & $0.77(0.53-1.13)$ & $0.73(0.30-1.79)$ & $0.94(0.66-1.35)$ & $0.17(0.05-0.63)$ \\
\hline No & 1 & 1 & $\mathrm{I}$ & 1 \\
\hline \multicolumn{5}{|l|}{ Justifies wife beating } \\
\hline Yes & $0.88(0.67-1.16)$ & $0.50(0.26-0.99)$ & $1.10(0.97-1.24)$ & $0.76(0.49-1.18)$ \\
\hline No & 1 & 1 & 1 & 1 \\
\hline \multicolumn{5}{|l|}{ Age at first marriage } \\
\hline$\leq 19$ years & $0.61(0.45-0.82)$ & $0.53(0.27-1.05)$ & $0.67(0.58-0.79)$ & $2.63(1.62-4.29)$ \\
\hline$\geq 20$ years & 1 & 1 & I & 1 \\
\hline \multicolumn{5}{|l|}{ Control over earnings } \\
\hline Yes & 1 & 1 & 1 & 1 \\
\hline No & I.I5 (0.69-I.89) & $0.93(0.34-2.53)$ & $1.32(1.09-1.58)$ & $0.94(0.53-1.68)$ \\
\hline \multicolumn{5}{|l|}{ Relationship inequality } \\
\hline \multicolumn{5}{|l|}{ Age inequality } \\
\hline Younger than partner & $0.85(0.36-2.04)$ & $0.58(0.11-3.14)$ & $1.24(0.9 \mid-1.68)$ & $1.09(0.5 \mathrm{I}-2.37)$ \\
\hline Same age as partner & 1 & 1 & $\mathrm{I}$ & 1 \\
\hline Older than partner & $0.47(0.15-1.53)$ & $0.35(0.06-2.21)$ & $1.07(0.69-1.66)$ & $0.47(0.16-1.39)$ \\
\hline \multicolumn{5}{|l|}{ Education inequality } \\
\hline Less educated than partner & $0.88(0.63-1.24)$ & $0.97(0.4 \mathrm{I}-2.29)$ & $1.15(0.99-1.32)$ & $1.34(0.84-2.13)$ \\
\hline Same education as partner & 1 & 1 & $\mathrm{I}$ & 1 \\
\hline More educated than partner & $0.50(0.34-0.74)$ & $1.46(0.56-3.79)$ & $1.15(0.95-1.39)$ & $1.38(0.69-2.78)$ \\
\hline \multicolumn{5}{|l|}{ Earning inequality } \\
\hline Earns less than partner & I.I5 (0.79-I.67) & $1.35(0.56-3.29)$ & $0.99(0.82-1.20)$ & $0.92(0.58-1.46)$ \\
\hline Earns the same as partner & 1 & 1 & 1 & 1 \\
\hline Earns more than partner & I.0I (0.6I-I.67) & $1.69(0.49-5.75)$ & $0.93(0.72-1.2 I)$ & $1.06(0.47-2.40)$ \\
\hline \multicolumn{5}{|l|}{ Age of respondent } \\
\hline $15-24$ years & $0.38(0.22-0.65)$ & $0.26(0.07-0.96)$ & $0.43(0.34-0.55)$ & $0.46(0.20-1.10)$ \\
\hline $25-34$ years & $0.69(0.47-1.02)$ & $0.45(0.18-1.09)$ & $0.59(0.49-0.73)$ & $0.60(0.29-1.22)$ \\
\hline$\geq 35$ years & 1 & $\mathrm{I}$ & 1 & $\mathrm{I}$ \\
\hline \multicolumn{5}{|l|}{ Highest educational level } \\
\hline No education & $0.11(0.06-0.23)$ & $0.19(0.07-0.54)$ & $0.42(0.36-0.50)$ & $0.42(0.25-0.70)$ \\
\hline Primary & $0.48(0.34-0.69)$ & $0.60(0.27-1.33)$ & $0.62(0.52-0.75)$ & $0.63(0.37-1.06)$ \\
\hline Secondary or higher & 1 & $\mathrm{I}$ & 1 & 1 \\
\hline \multicolumn{5}{|l|}{ Number of children ever born } \\
\hline $\mathrm{I}-2$ & 1 & 1 & 1 & 1 \\
\hline $3-4$ & $0.58(0.40-0.86)$ & $0.43(0.18-1.03)$ & $0.37(0.32-0.43)$ & $0.32(0.19-0.55)$ \\
\hline$\geq 5$ & $0.34(0.21-0.55)$ & $0.22(0.07-0.67)$ & $0.20(0.16-0.25)$ & $0.44(0.20-0.94)$ \\
\hline \multicolumn{5}{|l|}{ Place of residence } \\
\hline Urban & 1 & $\mathrm{I}$ & I & I \\
\hline Rural & $0.36(0.25-0.52)$ & $0.22(0.09-0.5 \mathrm{I})$ & $0.47(0.4 I-0.53)$ & $0.38(0.25-0.56)$ \\
\hline \multicolumn{5}{|l|}{ Wealth index } \\
\hline Poor & $0.36(0.25-0.5 I)$ & $0.22(0.09-0.54)$ & $0.26(0.22-0.31)$ & $0.35(0.22-0.57)$ \\
\hline Middle & $0.81(0.55-1.19)$ & $0.49(0.21-1.14)$ & $0.52(0.44-0.62)$ & $0.36(0.19-0.68)$ \\
\hline Rich & 1 & 1 & I & 1 \\
\hline
\end{tabular}

Note: Bold figures indicate statistical significance.

Abbreviations: $\mathrm{Cl}$, confidence interval; OR, odds ratio.

(OR: 0.50 ; 95\% CI: 0.34-0.74) compared to those with the same education as their partner. The likelihood of access to institutional delivery was significantly higher for women who were $\leq 19$ years at first marriage in Nepal (OR: 2.63; 95\% CI: 1.62-4.29) compared to those who were married at $\geq 20$ years, and for women who did not have control over earnings in India (OR: 1.32; 95\% CI: 1.09-1.58) compared to having control over earnings. Women who were aged 15-24 years old in Namibia (OR: 0.26 ; 95\% CI: 0.07-0.96) and India (OR: 0.43 ; 95\% CI: $0.34-0.55$ ) and $25-34$ years 
Table 5 Odds ratios and $95 \%$ confidence intervals for the association between measures of gender inequities and women's access to maternal tetanus vaccination during pregnancy

\begin{tabular}{|c|c|c|c|c|}
\hline \multirow[t]{3}{*}{ Variables } & \multicolumn{4}{|l|}{ Countries } \\
\hline & Kenya & Namibia & India & Nepal \\
\hline & $\begin{array}{l}\text { Adjusted } \\
\text { OR }(95 \% \mathrm{Cl})\end{array}$ & $\begin{array}{l}\text { Adjusted } \\
\text { OR }(95 \% \mathrm{Cl})\end{array}$ & $\begin{array}{l}\text { Adjusted } \\
\text { OR }(95 \% \mathrm{Cl})\end{array}$ & $\begin{array}{l}\text { Adjusted } \\
\text { OR }(95 \% \mathrm{Cl})\end{array}$ \\
\hline \multicolumn{5}{|l|}{ Gender inequities } \\
\hline \multicolumn{5}{|l|}{ Decision-making autonomy } \\
\hline Yes & 1 & I & I & I \\
\hline No & $0.93(0.11-7.84)$ & $1.87(0.73-4.78)$ & $\mathrm{I} .03(0.8 \mathrm{I}-\mathrm{I} .32)$ & $0.83(0.5 \mathrm{I}-1.34)$ \\
\hline \multicolumn{5}{|l|}{ Husband has other wives } \\
\hline Yes & $0.98(0.60-1.59)$ & $1.13(0.69-1.84)$ & $0.82(0.57-1.19)$ & $0.42(0.22-0.80)$ \\
\hline No & 1 & 1 & $\mathrm{I}+1$ & 1 \\
\hline \multicolumn{5}{|l|}{ Justifies wife beating } \\
\hline Yes & $1.00(0.68-1.48)$ & $1.10(0.74-1.65)$ & $1.05(0.92-1.20)$ & $0.74(0.5 \mathrm{I}-\mathrm{I} .05)$ \\
\hline No & 1 & 1 & $\mathrm{I}$ & $\mathrm{I}$ \\
\hline \multicolumn{5}{|l|}{ Age at first marriage } \\
\hline$\leq 19$ years & $0.96(0.62-1.47)$ & $1.30(0.87-1.93)$ & $0.93(0.76-1.15)$ & $\mathrm{I} .60(0.94-2.7 \mathrm{I})$ \\
\hline$\geq 20$ years & 1 & 1 & $\mathrm{I}$ & 1 \\
\hline \multicolumn{5}{|l|}{ Control over earnings } \\
\hline Yes & 1 & I & I & I \\
\hline No & $1.19(0.59-2.42)$ & $0.79(0.44-1.4 I)$ & $0.89(0.73-1.09)$ & $0.99(0.6 \mathrm{I}-\mathrm{I} .65)$ \\
\hline \multicolumn{5}{|l|}{ Relationship inequality } \\
\hline \multicolumn{5}{|l|}{ Age inequality } \\
\hline Younger than partner & $0.92(0.31-2.69)$ & $\mathrm{I} .03(0.57-1.85)$ & $1.14(0.77-1.68)$ & $1.72(0.86-3.44)$ \\
\hline Same age as partner & 1 & $\mathrm{I}$ & $\mathrm{I}+1$ & 1 \\
\hline Older than partner & $0.59(0.14-2.50)$ & $\mathrm{I} .07(0.5 \mathrm{I}-2.2 \mathrm{I})$ & $0.84(0.49-1.42)$ & $0.75(0.3 \mid-1.79)$ \\
\hline \multicolumn{5}{|l|}{ Education inequality } \\
\hline Less educated than partner & $1.07(0.69-1.66)$ & $1.28(0.82-1.98)$ & $1.33(1.15-1.55)$ & $1.37(0.95-1.97)$ \\
\hline Same education as partner & 1 & 1 & 1 & 1 \\
\hline More educated than partner & $0.80(0.48-1.35)$ & $1.02(0.65-1.59)$ & I.II (0.84-I.47) & $0.72(0.34-1.53)$ \\
\hline \multicolumn{5}{|l|}{ Earning inequality } \\
\hline Earns less than partner & $0.97(0.58-1.64)$ & $0.97(0.58-1.64)$ & $1.57(1.28-1.92)$ & $1.74(1.16-2.62)$ \\
\hline Earns the same as partner & 1 & 1 & 1 & 1 \\
\hline Earns more than partner & $0.85(0.43-1.66)$ & $1.38(0.73-2.63)$ & $1.54(1.14-2.07)$ & $0.74(0.35-1.58)$ \\
\hline \multicolumn{5}{|l|}{ Age of respondent } \\
\hline 15-24 years & I.3I (0.64-2.69) & $2.84(1.42-5.68)$ & $1.19(0.91-1.55)$ & $1.35(0.68-2.7 \mathrm{I})$ \\
\hline $25-34$ years & $1.15(0.72-1.82)$ & $1.29(0.83-1.99)$ & $1.39(1.14-1.68)$ & $1.54(0.92-2.57)$ \\
\hline$\geq 35$ years & 1 & 1 & I & 1 \\
\hline \multicolumn{5}{|l|}{ Highest educational level } \\
\hline No education & $0.23(0.11-0.47)$ & $1.19(0.55-2.55)$ & $0.35(0.27-0.46)$ & $0.34(0.18-0.63)$ \\
\hline Primary & $1.08(0.66-1.78)$ & $1.86(1.13-3.36)$ & $0.55(0.4 I-0.72)$ & $0.43(0.23-0.80)$ \\
\hline Secondary or higher & 1 & 1 & 1 & I \\
\hline \multicolumn{5}{|l|}{ Number of children ever born } \\
\hline $\mathrm{I}-2$ & I & I & I & I \\
\hline $3-4$ & $1.33(0.77-2.29)$ & $1.42(0.94-2.14)$ & $0.64(0.53-0.77)$ & $0.61(0.38-0.99)$ \\
\hline$\geq 5$ & $0.63(0.34-1.18)$ & $1.57(0.85-2.89)$ & $0.38(0.30-0.47)$ & $0.65(0.35-1.22)$ \\
\hline \multicolumn{5}{|l|}{ Place of residence } \\
\hline Urban & 1 & 1 & I & 1 \\
\hline Rural & I.II (0.67-1.83) & $0.92(0.6 \mathrm{I}-1.39)$ & $0.95(0.79-1.14)$ & $0.86(0.58-1.27)$ \\
\hline \multicolumn{5}{|l|}{ Wealth index } \\
\hline Poor & $1.28(0.73-2.24)$ & $0.99(0.55-1.8 I)$ & $0.3 \mathrm{I}(0.23-0.40)$ & $0.56(0.37-0.84)$ \\
\hline Middle & $1.22(0.67-2.24)$ & $1.47(0.91-2.39)$ & $0.61(0.46-0.80)$ & $1.06(0.62-1.80)$ \\
\hline Rich & 1 & I & I & I \\
\hline
\end{tabular}

Note: Bold figures indicate statistical significance.

Abbreviations: $\mathrm{Cl}$, confidence interval; $\mathrm{OR}$, odds ratio.

old in India (OR: 0.59; 95\% CI: 0.49-0.73) were less likely to access institutional delivery compared to those who were $\geq 35$ years, whilst women with no education (OR: 0.11; 95\% CI: $0.06-0.23$ ) or primary education (OR: 0.48 ; 95\% CI: 0.34-0.69) in Kenya, women with no education
(OR: 0.19; 95\% CI: 0.07-0.54) in Namibia, women with no education (OR: 0.42 ; 95\% CI: $0.36-0.50$ ) or primary education (OR: 0.62; 95\% CI: 0.52-0.75) in India, and women with no education (OR: $0.42 ; 95 \% \mathrm{CI}: 0.25-0.70)$ in Nepal were less likely to have access to institutional delivery 
compared to those with secondary or higher education. Women residing in rural areas in Kenya (OR: 0.36; 95\% CI: 0.25-0.52), Namibia (OR: 0.22; 95\% CI: 0.09-0.51), India (OR: 0.47; 95\% CI: 0.41-0.53), and Nepal (OR: 0.38; 95\% CI: $0.25-0.56)$ were less likely to have access to institutional delivery compared to those residing in urban areas. Living in households of poor wealth in Kenya (OR: 0.36 ; 95\% CI: 0.25-0.51), Namibia (OR: 0.22; 95\% CI: 0.09-0.54), India (OR: 0.26; 95\% CI: 0.22-0.31), and Nepal (OR: 0.35; 95\% CI: $0.22-0.57)$ and living in households of middle wealth in India (OR: 0.52; 95\% CI: 0.44-0.62) and Nepal (OR: 0.36; 95\% CI: 0.19-0.68) decreased the likelihood of accessing institutional delivery compared to those living in households of rich wealth (Table 4).

\section{Access to maternal tetanus vaccination during pregnancy}

Women in polygynous relationships in Nepal (OR: 0.42; 95\% CI: 0.22-0.80) were less likely to receive maternal tetanus vaccination during pregnancy compared to women in monogamous relationships. In contrast, the likelihood of receiving maternal tetanus vaccination during pregnancy was higher for women with less education than their partner in India (OR: 1.33 ; 95\% CI: 1.15-1.55) compared to having the same educational level as their partner, for women earning less than their partner in India (OR: $1.57 ; 95 \%$ CI: $1.28-1.92$ ) and in Nepal (OR: 1.74; 95\% CI: 1.16-2.62), and for women earning more than their partner in India (OR: 1.54 ; 95\% CI: 1.14-2.07) compared to earning the same as their partner.

Women aged 15-24 years in Namibia (OR: 2.84; 95\% CI: $1.42-5.68$ ) and 25-34 years in India (OR: 1.39; 95\% CI: 1.14-1.68) had a higher likelihood of receiving maternal tetanus vaccination during pregnancy. Women with no education in Kenya (OR: 0.23 ; 95\% CI: $0.11-0.47$ ), no education (OR: 0.35 ; 95\% CI: $0.27-0.46$ ) or primary education (OR: $0.55 ; 95 \% \mathrm{CI}: 0.41-0.72)$ in India, and no education (OR: 0.34 ; $95 \%$ CI: $0.18-0.63$ ) or primary education (OR: 0.43 ; 95\% CI: $0.23-0.80)$ in Nepal were less likely to receive a maternal tetanus vaccination during pregnancy compared to those with secondary or higher education, whereas having primary education in Namibia (OR: 1.86; 95\% CI: 1.13-3.36) was associated with a higher likelihood of receiving maternal tetanus vaccination during pregnancy compared to having secondary or higher education. Women who had three or four children in India (OR: 0.64; 95\% CI: 0.53-0.77) and Nepal (OR: 0.61 ; 95\% CI: $0.38-0.99$ ) and women who had five or more children in India (OR: 0.38 ; 95\% CI: 0.30-0.47) were less likely to receive maternal tetanus vaccination during pregnancy compared to women with one or two children. Finally, compared to women in rich households, women in households of poor (OR: 0.31 ; 95\% CI: $0.23-0.40)$ and middle wealth (OR: 0.61 ; 95\% CI: 0.46-0.80) in India, as well as those in households of poor wealth in Nepal (OR: 0.56 ; 95\% CI: $0.37-0.84$ ) were less likely to access maternal tetanus vaccination during pregnancy (Table 5).

\section{Discussion}

\section{Summary of findings}

Measures of gender inequities such as polygyny (husband having other wives), justifying wife beating, age at first marriage ( $\leq 19$ years), and education inequality (being less educated than partner) were differentially and significantly associated with the different measures of access to reproductive health care within the four countries.

\section{Comparison with previous studies Polygamy}

The women in polygynous relationships had lower access to institutional delivery and maternal tetanus vaccination during pregnancy in Nepal, consistent with findings from recent reports, ${ }^{39,40}$ which attribute this to the systematic gender and cultural discriminations deterring women from accessing modern reproductive health services in the form of early marriage, discrimination against daughters in access to health care, and restraining women's autonomy and physical mobility to seek health care for themselves; these are all reflective of a difference in men's and women's economic roles and power. ${ }^{41}$ Alternative explanations could be disadvantages arising from such factors as poor geographical accessibility, caste/ethnicity, and low income. ${ }^{40}$ These findings stress the need to change the patriarchal and discriminatory traditional value systems and practices in Nepal. The current findings are consistent with those in a study in SSA where women in monogamous relationships in Kenya and the Ivory Coast were found to seek more health care. ${ }^{42}$

\section{Justifying wife beating}

Women who considered wife beating justifiable were found to be less likely to have access to institutional delivery in Namibia. This may be due to lower status within the household, lower education, economic dependence on their male partners, and inexposure to a modern health care system, thus reflecting traditional gender role attitudes that place women in subordinate and dependent positions in relation to men. Coupled with the lower socioeconomic status of most of the respondents, it is plausible that the women lacked enough 
relationship and economic autonomy to demand access to reproductive health care. Similar findings have been reported in other studies. ${ }^{43}$

\section{Age at first marriage}

Findings in the association between early marriage ( $\leq 19$ years) and access to reproductive health care were mixed. Women who married at $\leq 19$ years were less likely to have access to institutional delivery in Kenya and India, which is consistent with another study, ${ }^{10}$ and may be due to hierarchies existing in families in which these women have little or no influence in decision making. ${ }^{36}$ Early marriage often curtails young women's educational opportunities,${ }^{44}$ and renders them less autonomous and incapable of asserting themselves in their marriage. Typically coinciding with early childbearing, it often results in lower access to reproductive health care, especially as these young brides may lack knowledge of sexual and reproductive matters. ${ }^{44}$ Conversely, marrying at $\leq 19$ years in Nepal was associated with increased access to skilled antenatal care and institutional delivery. This may be explained by early marriage being a societal norm in Nepal, ${ }^{45}$ evidenced by $40 \%$ of marriages involving girls 15 years of age in Nepal. ${ }^{46}$ The resulting early pregnancies are associated with an increased need for reproductive health care among women in this age group. Moreover, the decision to get girls married at an early age is also a reflection of underlying patriarchal values in Nepal. ${ }^{47}$

\section{Control over earnings}

That women lacking control over earnings had higher access to institutional delivery in India is counterintuitive and contrasts with findings in another study. ${ }^{35}$ Cultural norms and family dynamics are known to limit women's ability to exercise control over earnings/savings; however, women's participation in microcredit and savings schemes, which are socially accepted strategies to save in India, may enable them protect their savings from husbands and other relatives. ${ }^{48}$ Women may also choose individual savings programs that allow them to keep knowledge or details of their savings to themselves, so as to avoid being subjected to pressure from others, including their spouse. ${ }^{49}$

\section{Relationship inequalities}

The correlation between relationship inequalities in age, education, and earnings and different aspects of women's reproductive health care were also conflicting. That women in India who earned more than their spouse had higher access to maternal tetanus vaccination during pregnancy is as expected, and may result from the women's greater socioeconomic status and financial autonomy within the household, consistent with findings in other studies. ${ }^{50,51}$ The decreased access to reproductive health care associated with women being younger or less educated than their spouse may also be a reflection of their low status within the relationship and their economic dependence on their partners. In contrast, however, women who were more educated than their spouse being less likely to access reproductive health care or being less educated and more likely to access reproductive health care are indicative of the variation and complexity of manifestations of gender inequities within different social contexts. These associations point to gaps in the extant literature and the need for further research into the role of social inequalities within the household on different health care outcomes. It is presumable that lower access to reproductive health care among women who are more educated than their spouse may be related to these women being working women with time constraints reducing their use of reproductive health care; this is consistent with findings from another study, ${ }^{35}$ and may be of interest to those working with interventions aimed at increasing employment opportunities, as this may not always generate the expected results of either improving intrahousehold equality or access to health care.

\section{Demographic and socioeconomic factors}

The lower likelihood of accessing skilled antenatal care in Namibia, and institutional delivery in the four countries for women aged 15-24 years (and women aged 25-34 years in India) is in agreement with results from other studies, ${ }^{10,36}$ and could be attributed to older women having more decision-making autonomy. Moreover, younger women tend to have lesser say in household decisions, which are commonly made by either their partners or mothers-in-law within these contexts. Young age may also correlate with lower educational levels, and therefore less knowledge about maternal health care. ${ }^{45}$ The exception was a higher likelihood of access to maternal tetanus vaccination during pregnancy among women aged 15-24 years in Namibia and women aged 25-34 years in India, which may be associated with women aged $<35$ years having greater access to maternal health care as a result of their increased fertility rates, ${ }^{52}$ and higher parity. ${ }^{10,53}$

It was found that educational attainment of women was positively correlated with access to reproductive health care, in line with a previous study; ${ }^{35,53}$ reasons include increased awareness of the benefits of maternal health care among more educated women, greater influence over decisions regarding 
their own health, and better communication skills. ${ }^{10,35,36,53}$ The exception in this study being higher access to maternal tetanus vaccination during pregnancy among women with primary education compared to those with secondary or higher education in Namibia, which, as earlier stated, may be associated with the possibility of wanting to pursue their careers, hence decreased fertility, parity, and lower access to maternal tetanus vaccination among women with higher education.

Having three or more children was generally associated with less likelihood of accessing health care among women in all the countries. While it is unclear why women with three or more children are less likely to seek reproductive health care than women with two children or less, the results appear to be consistent with most studies done elsewhere which attribute this to increased confidence in home birth and the belief that modern health care is either not very necessary, or to high costs or negative experiences from previous pregnancies and births coupled with greater demands in terms of time, resources, and child care, ${ }^{52}$ and the poor quality of health care facilities. ${ }^{35}$ Women tend to use reproductive health care for their first child than later children, ${ }^{54}$ due to first pregnancies being more associated with difficulties during labor and delivery compared to high order pregnancies. Thus, low parity women are more motivated to deliver in medical facilities than high parity women.

This study also found a negative correlation between rural residence and access to skilled antenatal care (India and Nepal), as well as institutional delivery in all the countries; this is in line with existing literature, ${ }^{10,36,44,52,55,56}$ which have shown that urban residence confers an advantage in access to maternal health care. Contributing factors include longer travelling distances, time, and costs to reproductive health care services for women in many rural areas, often fewer health care facilities, and a general lack of skilled health care workers. In addition, women living in rural settings also tend to be less educated and of lower socioeconomic status, which could further limit their access to health care. Finally, household wealth index was found to be a significant predictor of access to maternal health care in these countries, which is an expected finding given that the majority of health care services in these countries are financed through out-of-pocket expenditure. ${ }^{57,58}$ Previous studies have shown that in addition to being indicative of the ability to pay for services, ${ }^{10,52,55,59}$ better wealth status may also reflect a greater perceived need of such skilled health care services. ${ }^{35,53,60}$ Only one measure of gender inequity - decision-making autonomy - was not significantly associated with women's access to reproductive health care; this may have resulted from differences in understanding the questions relating to these variables as well as underreporting among the women.

\section{Strengths and limitations}

The strengths of this study include: (1) the large sample size of DHS surveys; (2) the surveys being nationally representative and enabling the generalization of the results within and across countries; and (3) similar definitions of variables across countries, which increased comparability across countries. The limitations of this study include: (1) the cross-sectional nature of the data did not enable causal inference to be drawn from findings in this study; (2) household income or expenditure, which are commonly used measures for wealth, are not routinely collected in DHS surveys. The assets-based wealth index used in this study is only a proxy indicator for household economic status, which may not always produce results similar to those obtained from direct assessments of income and expenditure where such data are available or can be reliably collected; (3) the main outcome variable in this study was "access to reproductive health care" which was a proxy for "use" of health care. However, use of health care is also affected by availability, affordability, and acceptability of the services, and these need to be accounted for before any conclusions can be drawn regarding the use of health care; and (4) gender-based violence was not analyzed in this study, as data was not available for this variable in the DHS surveys for Namibia and India. Given the variations in cultural norms, health care systems, and sociodemographic characteristics both within and between the four countries, the current findings indicate that measures of gender inequities cannot be generalized to fit the different contexts.

\section{Conclusion and policy implications}

Dimensions of gender inequities (with the exception of decision-making autonomy) were significant predictors of access to reproductive health care. Findings highlight the role of patriarchal and discriminatory traditional value systems and practices that place women and girls in subordinate positions to men and boys within different social contexts, and the urgent need for concerted and sustained efforts to change these harmful traditional values if several of these countries are to meet MDG-5.

\section{Disclosure}

The authors report no conflicts of interest in this work. 


\section{References}

1. World Health Organization. MDG 5: improve maternal health. 2011. Available from: http://www.who.int/topics/millennium_development_ goals/maternal_health/en/index.html. Accessed May 3, 2011.

2. World Health Organization Regional Office for Africa. Reducing Maternal Deaths: The Challenge of the New Millennium in the African Region. Brazzaville; World Health Organization Regional Office for Africa; 2002

3. Stevens RD. Safe motherhood: an insight into maternal mortality in the developing world. Health Millions. 2000;26(3):34-37.

4. World Health Organization. Maternal mortality in 2005: estimates developed by WHO, UNICEF, UNFPA, and the World Bank. 2007 Available from: http://www.who.int/maternal_child_adolescent/ documents/9789241596213/en/index.html. Accessed May 2, 2011.

5. Mahler H. The safe motherhood initiative: a call to action. Lancet. 1987;1 (8534):668-670.

6. Starrs AM. Safe motherhood initiative: 20 years and counting. Lancet. 2006;368(9542):1130-1132.

7. United Nations. The millenium development goals. 2010. Available from: http://www.un.org/millenniumgoals/bkgd.shtml. Accessed April 23, 2011.

8. Campbell OM, Graham WJ. Strategies for reducing maternal mortality: getting on with what works. Lancet. 2006;368(9543) 1284-1299.

9. Bulatao RA, Ross JA. Which health services reduce maternal mortality? Evidence from ratings of maternal health services. Trop Med Int Health 2003;8(8):710-721.

10. Simkhada B, Teijlingen ER, Porter M, Simkhada P. Factors affecting the utilization of antenatal care in developing countries: systematic review of the literature. J Adv Nurs. 2008;61(3):244-260.

11. Carroli G, Rooney C, Villar J. How effective is antenatal care in preventing maternal mortality and serious morbidity? An overview of the evidence. Paediatr Perinat Epidemiol. 2001;15 Suppl 1:1-42.

12. Koblinsky MA, Campbell O, Heichelheim J. Organizing delivery care: what works for safe motherhood? Bull World Health Organ. 1999;77(5): 399-406.

13. Adegoke AA, van den Broek N. Skilled birth attendance-lessons learnt. BJOG. 2009;116 Suppl 1:33-40.

14. Bullough C, Meda N, Makowiecka K, Ronsmans C, Achadi E, Hussein J. Current strategies for the reduction of maternal mortality. BJOG. 2005;112(9):1180-1188.

15. Ronsmans C, Graham WJ. Maternal mortality: who, when, where, and why. Lancet. 2006;368(9542):1189-1200.

16. World Health Organization. Trends in maternal mortality: 1990 to 2008. Estimates developed by WHO, UNICEF, UNFPA, and The World Bank. 2010. Available from: http://www.unfpa.org/public/ home/publications/ pid/6598. Accessed April 16, 2011.

17. Rogo KO, Oucho J, Mwalali P. Maternal mortality. In: Jamison DT, Feachem RG, Makgoba MW, et al., editors. Disease and Mortality in sub-Saharan Africa. 2nd ed. Washington, DC: The World Bank; 2006:223-236.

18. Rabkin M, El-Sadr WM, Mugyenyi P, Ramatlapeng MK, De Cock KM. Lessons from Africa. J Acquir Immune Defic Syndr. 2010;55 Suppl 2 S141-S143.

19. Khan KS, Wojdyla D, Say L, Gulmezoglu AM, Van Look PF. WHO analysis of causes of maternal death: a systematic review. Lancet. 2006;367(9516):1066-1074.

20. Hogan MC, Foreman KJ, Naghavi M, et al. Maternal mortality for 181 countries, 1980-2008: a systematic analysis of progress towards Millennium Development Goal 5. Lancet. 2010;375(9726): 1609-1623.

21. Buor D, Bream K. An analysis of the determinants of maternal mortality in sub-Saharan Africa. $J$ Womens Health (Larchmt) 2004;13(8):926-938.

22. McTavish S, Moore S, Harper S, Lynch J. National female literacy, individual socio-economic status, and maternal healthcare use in subSaharan Africa. Soc Sci Med. 2010;71(11):1958-1963.
23. Asian-Pacific Resource and Research Centre for Women. MDG5 in Asia: progress, gaps and challenges 2000-2010. 2010. Available from: http:// www.mdg5watch.org/Regional/MDG5RegionalBrief.pdf. Accessed April 16, 2011.

24. Amery J. Safe motherhood case studies: learning from South Asia. J Health Popul Nutr. 2009;27(2):87-88.

25. Thatte N, Mullany LC, Khatry SK, Katz J, Tielsch JM, Darmstadt GL. Traditional birth attendants in rural Nepal: knowledge, attitudes and practices about maternal and newborn health. Glob Public Health 2009;4(6):600-617.

26. Kumar S. Reducing maternal mortality in India: policy, equity, and quality issues. Indian J Public Health. 2010;54(2):57-64.

27. Ridgeway CL, Correll SJ. Motherhood as a status characteristic. J Soc Issues. 2004;60(4):683-700.

28. Kishor $\mathrm{S}$. The heavy burden of a silent scourge: domestic violence. Rev Panam Salud Publica. 2005;17(2):77-78.

29. Whitehead M. The concepts and principles of equity and health. Int $J$ Health Serv. 1992;22(3):429-445.

30. United Nations Population Fund. Gender equality: a cornerstone of development. 2011. Available from: http://www.unfpa.org/gender/. Accessed April 16, 2011.

31. Kabeer N. Gender equality and women's empowerment: a critical analysis of the third Millennium Development Goal. Gend Dev. 2005;13(1):13-24.

32. Sen A. The many faces of gender inequality. New Repub. September 17, 2001:35-40

33. World Health Organization. Women and health: today's evidence tomorrow's agenda. 2009. Available from: http://www.who.int/ gender/documents/9789241563857/en/index.html. Accessed April 16, 2011.

34. Bronfenbrenner U. Toward an experimental ecology of human development. Am Psychol. 1977;32(7):513-531.

35. Furuta M, Salway S. Women's position within the household as a determinant of maternal healthcare use in Nepal. Int Fam Plan Perspect. 2006;32(1):17-27.

36. Gabrysch S, Campbell OM. Still too far to walk: literature review of the determinants of delivery service use. BMC Pregnancy Childbirth. 2009;9:34.

37. Heise L, Ellsberg M, Gottemoeller M. Ending violence against women. Popul Rep L. 1999;27(11):1-43.

38. Linos N, Khawaja M, Al-Nsour M. Women's autonomy and support for wife beating: findings from a population-based survey in Jordan Violence Vict. 2010;25(3):409-419.

39. Deuba AR, Rana PS. A Study on the Psycho-Social Impacts of Violence Against Women and Girls With Special Focus on Rape, Incest, and Polygamy. Lalitpur: SAATHI Organization; 2001.

40. UNFPA. Gender equality and empowerment of women in Nepal; 2007. Available from: http://nepal.unfpa.org/pdf/Gender\%20Equality.pdf

41. Desai S. Gender Inequalities and Demographic Behavior in India. New York, NY: The Population Research Council; 2007.

42. Stephenson R, Baschieri A, Clements S, Hennink M, Madise N. Contextual influences on the use of health facilities for childbirth in Africa. Am J Public Health. 2006;96(1):84-93.

43. Jensen R, Thornton R. Early female marriage in the developing world. Gend Dev. 2003;11(2):9-19.

44. Santhya KG, Ram U, Acharya R, Jejeebhoy SJ, Ram F, Singh A. Associations between early marriage and young women's marital and reproductive health outcomes: evidence from India. Int Perspect Sex Reprod Health. 2010;36(3):132-139.

45. Singh S, Samara R. Early marriage among women in developing countries. Int Fam Plan Perspect. 1996;22(4):148-157.

46. Gazmararian JA, Lazorick S, Spitz AM, Ballard TJ, Saltzman LE, Marks JS. Prevalence of violence against pregnant women. JAMA. 1996;275(24):1915-1920.

47. Haywood RF. Breaking the Earthenware Jar: Lesson From South Asia to End Violence Against Women and Girls. Kathmandu: UNICEF Regional Office for South Asia; 2000. 
48. Gugerty MK. You can't save alone: commitment and rotating savings and credit associations in Kenya. Econ Dev Cult Change. 2007;55(2):251-282.

49. Fletschner D, Kenney L. Rural Women's Access to Financial Services: Credit, Savings, and Insurance. Rome: Food and Agriculture Organization of the United Nations; 2011.

50. Grown C, Gupta GR, Pande R. Taking action to improve women's health through gender equality and women's empowerment. Lancet. 2005;365(9458):541-543.

51. Freedman L. Strategic advocacy and maternal mortality: moving targets and the millennium development goals. Gend Dev. 2003;11(1): 97-108.

52. Neupane S, Doku DT. Determinants of time of start of prenatal care and number of prenatal care visits during pregnancy among Nepalese women. J Community Health. November 30, 2011. [Epub ahead of print.]

53. Ochako R, Fotso JC, Ikamari L, Khasakhala A. Utilization of maternal health services among young women in Kenya: insights from the Kenya Demographic and Health Survey, 2003. BMC Pregnancy Childbirth. 2011;11:1.

54. Celik Y, Hotchkiss DR. The socio-economic determinants of maternal healthcare utilization in Turkey. Soc Sci Med. 2000;50(12): 1797-1806.
55. Zere E, Tumusiime P, Walker O, Kirigia J, Mwikisa C, Mbeeli T. Inequities in utilization of maternal health interventions in Namibia: implications for progress towards MDG 5 targets. Int J Equity Health. 2010;9:16.

56. Say L, Raine R. A systematic review of inequalities in the use of maternal healthcare in developing countries: examining the scale of the problem and the importance of context. Bull World Health Organ. 2007;85(10):812-819.

57. Ministry of Health and Family Welfare, India. National health accounts, India: 2001-2002. December 2005. Available from: http://siteresources. worldbank.org/INTHSD/Resources/376278-1261143298590/66601791280173228245/India2001_02.pdf. Accessed May 3, 2011.

58. Ministry of Health and Population, Nepal. National health accounts: 2003/4 to 2005/6. July 2009. Available from: http://www.who.int/ nha/country/npl/Nepal_NHA_2003_4-2005_6.pdf. Accessed May 3, 2011 .

59. Navaneetham K, Dharmalingam A. Utilization of maternal healthcare services in Southern India. Soc Sci Med. 2002;55(10):1849-1869.

60. Woldemicael G. Do women with higher autonomy seek more maternal healthcare? Evidence from Eritrea and Ethiopia. Health Care Women Int. 2010;31(7):599-620.
International Journal of Women's Health

\section{Publish your work in this journal}

The International Journal of Women's Health is an international, peerreviewed open-access journal publishing original research, reports, reviews and commentaries on all aspects of women's healthcare including gynecology, obstetrics, and breast cancer. Subject areas include: Chronic conditions (migraine headaches, arthritis, osteoporosis);

\section{Dovepress}

Endocrine and autoimmune syndromes; Sexual and reproductive health; Psychological and psychosocial conditions. The manuscript management system is completely online and includes a very quick and fair peer-review system. Visit http://www.dovepress.com/ testimonials.php to read real quotes from published authors. 